\title{
PROPERTIES OF LUSIN SETS WITH APPLICATIONS TO PROBABILITY
}

\author{
R. B. DARST 1
}

In this note we shall show that a Lusin set is so thin that it can be used to construct several illuminating examples for probability.

I. Notation. In this paper a Lusin set will be understood to be an uncountable subset $\Omega$ of the interval $I=[0,1]$ such that if $F$ is a first category subset of $I$, then $\Omega \cap F$ is, at most, a countable set or, equivalently, if $\left\{y_{i}\right\}$ is a countable dense subset of $I$ and $\left\{\epsilon_{i}\right\}$ is a sequence of positive numbers, then $\Omega-U N\left(y_{i}, \epsilon_{i}\right)$ is countable, where $N(y, \epsilon)$ $=I \cap(y-\epsilon / 2, y+\epsilon / 2)$. By a universally measurable set we shall mean a subset $E$ of $R^{1}$ such that if $\mu$ is a nonatomic probability measure on the Borel subsets $B_{0}$ of $R^{1}$, then $\mu_{*}(E)=\mu^{*}(E)$. A set $E$ is universally measurable if, and only if, every homeomorphism $\phi$ of $R^{1}$ carries $E$ onto a set $\phi(E)$ which is Lebesgue measurable. A universal null set is a universally measurable set which is mapped into a Lebesgue null set by all homeomorphisms. Since it imposes no real restriction to suppose that a probability measure on $\beta_{0}$ is nonatomic, henceforth we do so suppose. Finally, denote by $B$ the Borel subsets $\Omega \cap B, B \in B_{0}$, of $\Omega$, i.e., $\mathbb{B}=\bigotimes_{0} \mid \Omega$.

II. The Borel measurable images of a Lusin set $\Omega$ are universal null sets. Notice that Borel measurability has two possible interpretations in the preceding assertion.

(1) If $f$ is a $\Theta_{1}$-measurable function on $I$, then $f(\Omega)$ is a universal null set in $R^{1}$, where $B_{1}=B_{0} \mid I$.

(2) If $f$ is a $B$-measurable function on $\Omega$, then $f(\Omega)$ is a universal null set.

For continuous $f$ these statements have long been verified (e.g. $[3$, p. 528]); but, for Borel measurable $f$ they seem to have gone unnoticed. Toward their verification let us first recall [3, pp. 399-400] that there is a first category subset $P$ of $I$ such that $f_{1}=f \mid I_{1}$ is continuous where $I_{1}=I-P$. Let $\left\{y_{i}\right\}$ be a countable dense subset of $I_{1}$ and $\epsilon>0$. Then $\left\{y_{i}\right\}$ is dense in $\Omega$ and, recalling that $\mu$ is nonatomic, there is a sequence $\epsilon_{i}$ of positive numbers such that $\mu\left(f_{1}\left(Q_{i}\right)\right)<\epsilon \cdot 2^{-i}$ where $Q_{i}=I_{1} \cap N\left(y_{i}, \epsilon_{i}\right)$. Let $Q=\cup_{i} Q_{i}$ and $P_{1}=I_{1}-Q$. The set $F=P \cup P_{1}$ is seen to be a first category subset of $I$ and, hence, $F \cap \Omega$

Received bv the editors June 21, 1967 and, in revised form October, 20, 1967.

1 This author is partially supported by an NSF grant. 
is countable which implies that $\mu(f(F \cap \Omega))=0$. Moreover, since $\Omega-F \subset Q, f_{1}\left(Q_{i}\right)=f\left(Q_{i}\right)$, and $\mu\left(\cup f_{1}\left(Q_{i}\right)\right) \leqq \sum \mu\left(f_{1}\left(Q_{i}\right)\right)<\epsilon$ we have $\mu(f(\Omega-F))=0$ and assertion (1) is established. Although minor changes in notation will yield (2), perhaps it is better to put them down: There is a first category subset $P$ of $\Omega([3$, pp. 399-400]) such that $f_{1}=f \mid I_{1}$ is continuous where $I_{1}=\Omega-P$. Let $\left\{y_{i}\right\}$ be a countable dense subset of $I_{1}, \epsilon>0$, and $\left\{\epsilon_{i}\right\}$ satisfy $\mu\left(f_{1}\left(Q_{i}\right)\right)<\epsilon \cdot 2^{-i}$, where $Q_{i}=I_{1} \cap N\left(y_{i}, \epsilon_{i}\right)$. As before the set $F=P \cup P_{1}$ is a first category subset of $\Omega$ where $Q=\cup Q_{i}$ and $P_{1}=I_{1}-Q$. Hence [3, pp. 525-526] $F$ is a countable set and (2) obtains.

III. Applications to probability. Recall [2] that a $D$-space is a measurable space $(X, \mathcal{F})$ such that (i) $\mathcal{F}$ is a separable sigma algebra of subsets of $X$ and (ii) if $f$ is a $F$-measurable function then $f(X)$ is a universally measurable set. The following characterization of a $D$ space has been established in [2]: $(X, F)$ is a $D$-space if, and only if, $(X, Q, \mu)$ is perfect (i.e., if $f$ is $Q$-measurable, $A \subset R^{1}$, and $f^{-1}(A) \in Q$, then there exists a Borel subset $B$ of $A$ such that $\left.\mu\left(f^{-1}(B)\right)=\mu\left(f^{-1}(A)\right)\right)$ whenever $Q$ is a separable subsigma algebra of $\mathcal{F}$ and $\mu$ is a probability measure on $a$. If $f(X)$ is an analytic set for each $\mathcal{F}$-measurable $f$, then $(X, \mathcal{F})$ is called a Lusin space $[1]$.

We shall verify the following statements which settle questions raised or implied in [1] and [2].

(3) Not every $D$-space is a Lusin space.

(4) The space $(\Omega, B)$ is a $D$-space but not a Lusin space.

(5) The identity function $j: j(x)=x, x \in \Omega$, is a $B$-measurable function whose range is not an analytic set.

(6) If $\nu$ is a probability measure on a separable subsigma algebra $\mathcal{F}$ of $B$, then $\nu$ is the restriction to $\mathcal{F}$ of a probability measure on $B$.

(7) All probability measures on $B$ are atomic. If $f$ is a $B$-measurable function on $\Omega$, then it follows from (2) that $f(\Omega)$ is a universal null set. Because an uncountable analytic set contains a perfect set and as a result, supports a non atomic probability measure, $f(\Omega)$ contains no uncountable analytic set. In particular, the range of the identity function $j$ is not an analytic set. Hence $(\Omega, B)$ is a $D$-space which is not a Lusin space and (3)-(5) are established. Turning now to (6) and (7), suppose that $\mu$ is a nonatomic probability measure on $\mathcal{F}$. Let $\{E(0), E(1)\}$ be a partition of $\Omega$ into two sets of $\mu$-measure $2^{-1}$, and let $\left\{E\left(i_{1}, \cdots, i_{k}, 0\right), E\left(i_{1}, \cdots, i_{k}, 1\right)\right\}$ be a partition of $E\left(i_{1}, \cdots, i_{k}\right)$ into two sets of $\mu$-measure $2^{-(k+1)}$. Let $f_{k}$ be defined on $\Omega$ by $f_{k}(x)=\sum_{j \leq k} i_{j}\left(3^{-j}\right)$ if $x \in E\left(i_{1}, \cdots, i_{k}\right)$ and, finally, let $f(x)$ $=\lim _{k} f_{k}(x)$. Then $f$ is a $\mathcal{F}$-measurable function on $\Omega$ and it follows 
from [2, Lemma] that there is a $F$-measurable set $\Omega_{0}$ such that $\mu\left(\Omega_{0}\right)=1$ and $f\left(\Omega_{0}\right)$ is a Borel set. Because $f$ is also $\beta$-measurable, $f(\Omega)$ is a universal null set. Hence $f\left(\Omega_{0}\right)$, being a subset of a universal null set and also a Borel set, must be countable. Let $\left\{t_{j}\right\}$ be an enumeration of $f\left(\Omega_{0}\right)$. Notice that $f\left(E\left(i_{1}, \cdots, i_{k}\right)\right) \cap f\left(E\left(j_{1}, \cdots, j_{k}\right)\right) \neq \varnothing$ if, and only if, $i_{p}=j_{p}, p \leqq k$. Let $E_{j}=E\left(i_{1}^{j}, \cdots, i_{j+1}^{\jmath}\right) \supset f^{-1}\left(t_{j}\right)$ and let $E=\cup_{j} E_{j}$. Then $\mu(E) \leqq 2^{-1}$ and $f(\Omega-E) \cap f\left(\Omega_{0}\right)=\varnothing$. This contradiction establishes that there are no nonatomic probability measures on $\mathcal{F}$. The decomposition of a probability measure $\nu$ on $\mathcal{F}$ into its atomic and nonatomic parts represents $\nu$ as a convex combination of an atomic and a nonatomic probability measure. Since the nonatomic component of $\nu$ is zero, $\nu$ is atomic. Moreover, elementary measure theory tells us that each atom can be associated with a point of $\Omega$ : Suppose that $\mathcal{F}$ is generated by the sequence $\left\{F_{j}\right\}$ of $B$-measurable sets and that $\nu=\sum x_{i} \nu_{i}$, where $x_{i}>0, \sum x_{i}=1$, and the $\nu_{i}$ 's are mutually singular two-valued probability measures on $\mathcal{F}$. Let $p_{i} \in \bigcap_{j}\left\{F_{j}, \nu_{i}\left(F_{j}\right)=1\right\}$ and let $\lambda$ be defined on $@$ by $\lambda(E)=\sum_{\left\{i ; p_{i} \in E\right\}} x_{i}$. Then $\lambda$ is an extension of $\nu$ to $B$.

IV. Borel images and measure. Recalling a construction of a Cantor set of positive Lebesgue measure in $I$ reminds us that any Borel probability measure on $I$ lies on a first category subset of $I$. This latter fact can be interpreted to say that if $\phi$ is a nondecreasing function from $I$ into $I$, then there is a first category subset $F$ of $I$ such that the Lebesgue measure $m(\phi(I-F))=0$. The technique applied to establish (1) permits us to claim results of which the following is an instance.

(8) If $b$ is Borel measurable on a separable metric space $M$, then there is a first category subset $F$ of $M$ such that $m(b(M-F))=0$.

\section{REFERENCES}

1. David Blackwell, On a class of probability spaces, Proc. Third Berkeley Sympos. on Math. Stat. and Prob., Vol. II, Univ. of Calif. Press, Berkeley, pp. 1-6.

2. Gopinath Kallianpur, $A$ note on perfect probability, Ann. Math. Statist. 30 (1959), 169-172.

3. Casimir Kuratowski, Topology, Vol. I, Academic Press, New York, 1966.

Purdue University 